\title{
Protocol for the Arterial Revascularisation Trial (ART). A randomised trial to compare survival following bilateral versus single internal mammary grafting in coronary revascularisation [ISRCTN46552265]
}

\author{
David P Taggart*1, Belinda Lees ${ }^{2}$, Alastair Gray33, Douglas G Altman ${ }^{4}$, \\ Marcus Flather ${ }^{2}$, Keith Channon ${ }^{5}$ and the ART Investigators
}

Address: ${ }^{1}$ Nuffield Dept of Surgery, University of Oxford, John Radcliffe Hospital, Oxford, OX3 9DU, UK, ${ }^{2}$ Clinical Trials and Evaluation Unit, Royal Brompton and Harefield NHS Trust, Sydney Street, London SW3 6NP, UK, ${ }^{3}$ Health Economics Research Centre, Institute of Health Sciences, Oxford OX3 7LF, UK, ${ }^{4}$ Cancer Research UK Medical Statistics Group, Centre for Statistics in Medicine, Wolfson College, University of Oxford, Linton Road, Oxford OX2 6UD, UK and ${ }^{5}$ Department of Cardiovascular Medicine, John Radcliffe Hospital, Headington, Oxford OX3 9DU, UK

Email: David P Taggart* - david.taggart@orh.nhs.uk; Belinda Lees - b.lees@rbh.nthames.nhs.uk; Alastair Gray - alastair.gray@dphpc.ox.ac.uk; Douglas G Altman - doug.altman@cancer.org.uk; Marcus Flather - m.flather@rbh.nthames.nhs.uk; Keith Channon - keith.channon@cardiov.ox.ac.uk; the ART Investigators - b.lees@rbh.nthames.nhs.uk

* Corresponding author

Published: 30 March 2006

Trials2006, 7:7 doi:10.1186/1745-6215-7-7

This article is available from: http://www.trialsjournal.com/content/7/I/7

(c) 2006Taggart et al; licensee BioMed Central Ltd.

This is an Open Access article distributed under the terms of the Creative Commons Attribution License (http://creativecommons.org/licenses/by/2.0), which permits unrestricted use, distribution, and reproduction in any medium, provided the original work is properly cited.
Received: 10 March 2006

Accepted: 30 March 2006

\begin{abstract}
Background: Standard coronary artery bypass graft surgery uses a single internal mammary artery and supplemental vein or radial artery grafts. Several observational studies have suggested a survival benefit with two internal mammary artery grafts compared to a single internal mammary artery graft, but this has not been tested in a randomised trial. The Arterial Revascularisation Trial is a Medical Research Council and British Heart Foundation funded, multi-centre international trial comparing single internal mammary artery grafting versus bilateral internal mammary artery grafting.

Methods/Design: Twenty centres in the UK, Australia, Poland and Brazil are planning to randomise 3000 coronary artery bypass graft surgery patients to single or bilateral internal mammary artery grafting. Supplemental grafts may be either saphenous vein or radial artery. Coronary artery bypass grafting can be performed as an on-pump or off-pump procedure. The primary outcome is survival at 10 years and secondary end-points include clinical events, quality of life and cost effectiveness. The effect of age, left ventricular function, diabetes, number of grafts, vein grafts and off-pump surgery are pre-specified subgroups.

Discussion: The Arterial Revascularisation Trial is one of the first randomised trials to evaluate the effects on survival and other clinical outcomes of single internal mammary artery grafting versus bilateral internal mammary artery grafting, and will help to establish the best approach for patients requiring coronary artery bypass graft surgery.
\end{abstract}




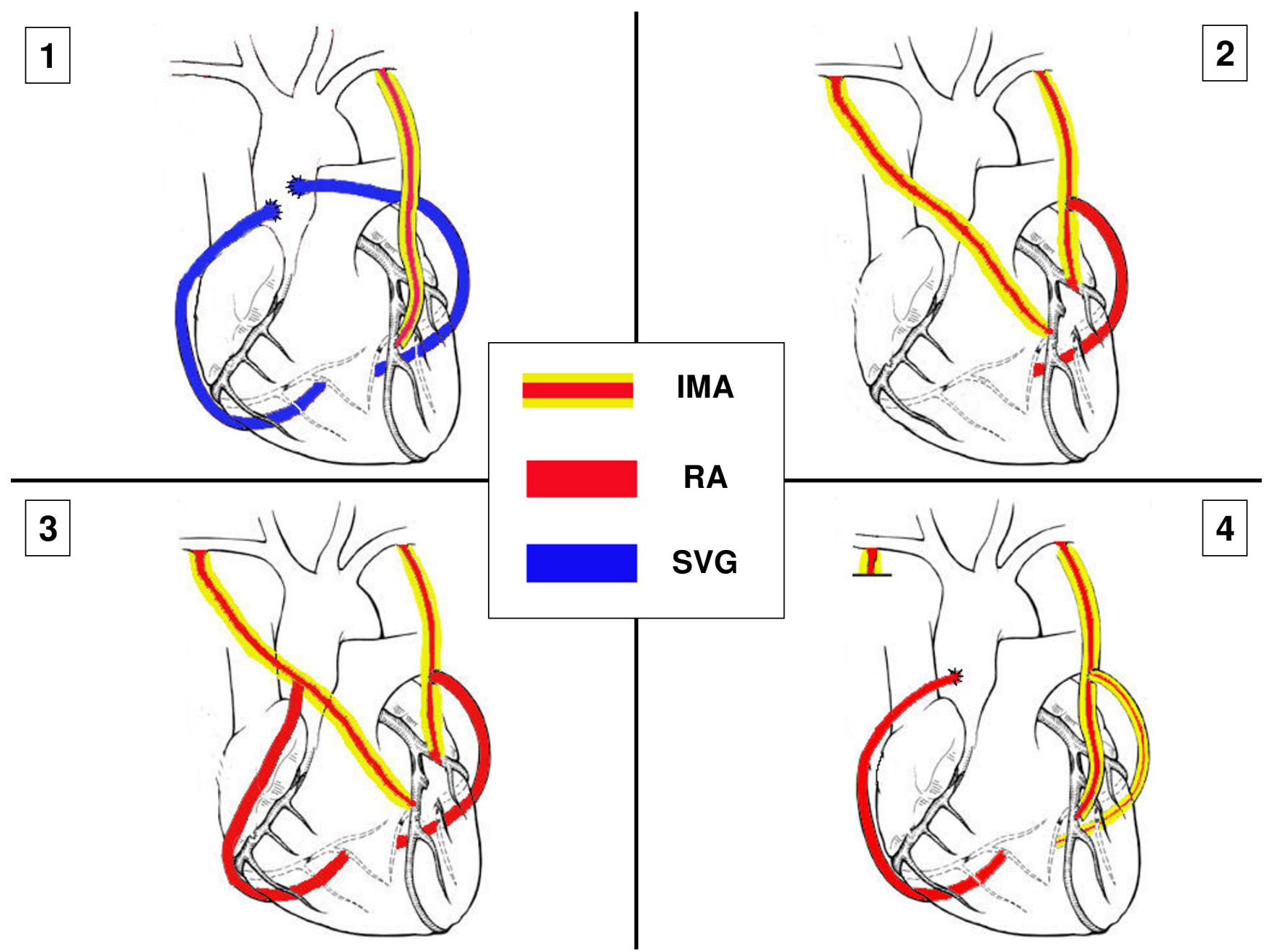

Figure I

Schematic drawing showing typical examples of: (I) Single internal mammary artery (SIMA) grafts and (2-4) Bilateral internal mammary artery (BIMA) grafts. Key: IMA = internal mammary artery; RA = radial artery; SVG = saphenous vein graft.

\section{Background}

Coronary artery bypass graft (CABG) surgery is the optimal therapy, prognostically and symptomatically, for multi-vessel ischaemic heart disease [1]. Worldwide, around 800,000 CABG are performed annually. Recognising the under provision of CABG in the UK, the National Service Framework (NSF) aims to increase numbers from 500 [2] to 750 per million of population. The requirement for CABG is also likely to increase because of a growing elderly population with coronary disease and because percutaneous interventions ultimately lead to an increased requirement for CABG [3].

Most CABG patients require three grafts and the standard operation uses a single internal mammary artery (SIMA) and supplemental vein or radial artery grafts (Figure 1). CABG provides excellent short and intermediate term out- comes but its long-term efficacy is limited by vein graft failure. Ten years after CABG around $1 / 2$ of vein grafts are blocked and of the remaining 50\% half are severely diseased [6] whereas up to $95 \%$ of internal mammary artery (IMA) conduits remain disease free. In addition to reducing long-term survival, vein graft failure significantly increases the risk of recurrent angina, late myocardial infarction and the need for further intervention [4-7]. Indeed, by 10 years after $C A B G>50 \%$ of patients have recurrent angina and up to $1 / 3$ may eventually require redo CABG [8-11]. Aspirin and statins [10,11] may improve vein graft patency but are unlikely to achieve the patency rates of IMA grafts.

As discussed below, bilateral IMA (BIMA) in comparison to SIMA grafts, may improve survival and reduce the need for redo CABG. However while BIMA grafting is common 


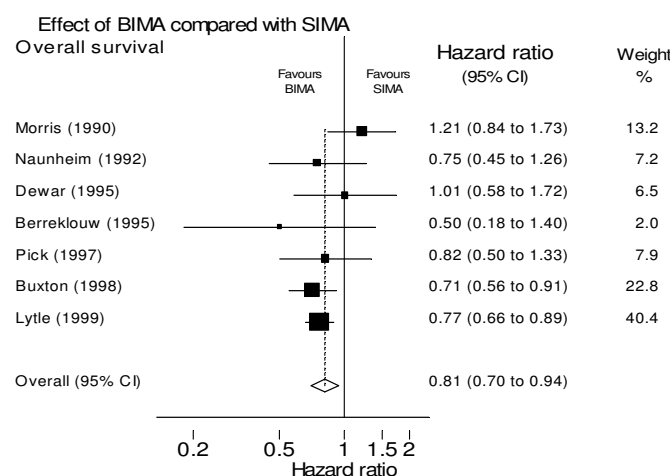

Figure 2

Survival following bilateral internal mammary artery (IMA) grafting compared to single IMA grafting [12].

in some centres in Europe, America, Australia and Japan, it still not widely used. For example, in 1998 15\% of UK CABG patients received two arterial grafts (and a significant proportion of these would have used a radial artery rather than a second IMA graft) [2]. The major reasons for not using BIMA grafts is because of no definitive evidence of benefits (there are no randomised trials) and the perceptions that it is technically more challenging, more prolonged and increases the risk of impaired wound healing. Given the number of CABG procedures currently performed in the UK and the aim of the NSF to increase these numbers, it is also important to obtain accurate information on the costs and cost-effectiveness of using BIMA versus SIMA grafts.

Nine studies, reviewed extensively in reference 12, have compared the influence of SIMA and BIMA grafts on survival and the need for redo surgery. Interpretation of individual studies is, however, complicated by lack of randomisation, small patient numbers and inadequate length or completeness of follow-up. Furthermore, as the use of BIMA grafts was initially confined to younger, lower risk patients, any long-term benefits were attributed to the inherently more favourable characteristics of these patients, obscuring any benefit of BIMA.

A recent systematic review was performed of those studies, meeting pre-specified criteria relating to patient selection, comparability of groups, outcome assessment, and completeness of follow-up, to determine if there are differences in survival, expressed as a pooled hazard ratio (HR), in patients receiving SIMA or BIMA [12] (Figure 2). The analysis included 15962 patients comprising 11269 SIMA and 4693 BIMA patients from seven studies that either matched or adjusted for age, sex, ventricular function and diabetes. The results suggested a survival advantage for BIMA grafts (HR for death $=0.81,95 \% \mathrm{CI}$ : 0.70 to 0.94 ) without any evidence of a detrimental effect, however there was very limited evidence relating to secondary endpoints including possible adverse consequences.

In the largest single study [13], of 8000 SIMA and 2000 BIMA patients, Lytle et al reported that the HR for death was decreased by $23 \%$ in the BIMA group at 12 years and the need for redo CABG reduced from $40 \%$ in the SIMA to $10 \%$ in the BIMA group.

In another observational study, published after the systematic review, Endo et al reported outcome in 688 SIMA and 443 BIMA patients [14]. The groups were similar regarding age and ventricular function but there were more diabetics in the BIMA group (18\% vs $13 \%)$ and females in the SIMA group (19\% vs $10 \%)$. At six years the combined incidence of death, myocardial infarction and redo CABG was lower in the BIMA group $(\mathrm{p}=0.06)$ and particularly in the 782 patients below 71 years and with an ejection fraction $>0.4$ (HR: $0.61 ; 95 \% \mathrm{CI}, 0.38$ to $0.98: \mathrm{p}=0.04)$. As vein graft failure increases markedly beyond seven years after CABG the authors suggest that the benefits of BIMA grafts are likely to increase with further follow-up.

Two studies reported no benefit of BIMA grafting $[15,16]$. Sergeant's study, however, had fewer than 100 BIMA patients with 10-year follow up and use of the second IMA was frequently suboptimal [15]. Kurlansky et al reported no survival difference at ten years in 261 women with BIMA grafts and a matched cohort with SIMA grafts [16]. However, $81 \%$ of the BIMA group vs $66 \%$ of the SIMA group had triple vessel disease $(\mathrm{p}<0.001)$ and only 120 patients in each group were available for comparison at ten years.

For optimal patency both IMA should be placed to the left sided arteries [17-19] (Figure 1). Patency of the right IMA is reduced if used as a free aortic graft [18] or placed to the right coronary artery [19] due to size discrepancy and eventual disease development at the crux.

Angiographic studies demonstrate markedly superior patency of BIMA grafts, compared to vein grafts, refuting the assertion that the superior patency of IMA grafts is due to better 'run-off' in the LAD territory. Patency rates for BIMA to various coronary arteries, are $98 \%$ at 7 days [14] and 95\% at two [20] and seven years [21]. Furthermore, offpump CABG (OPCAB) now makes CABG feasible in patients whose advanced age previously precluded CABG using cardiopulmonary bypass. A composite radial artery from one or both IMA, allows up to four grafts as an 'offpump' CABG, eliminating both cardiopulmonary bypass 
and aortic manipulation and minimizing the risk of cerebral injury [22].

Opposition to BIMA grafting is largely based on the perception of increased perioperative risk and especially sternal wound morbidity. There is consistent evidence, however, that the operative mortality of BIMA grafting at $1 \%-2 \%[12,13]$ is no higher, and may, in fact, be less than that of the standard operation because of a reduced risk of early graft failure.

Sternal dehiscence is a potential complication of BIMA grafts and particularly in diabetics. In reality, there is only a minimal increase in the risk of impaired wound healing in both non-diabetics [13,23-27] and diabetics [23-27] unless the patient is morbidly obese and/or has marked respiratory impairment [24]. The risk of impaired wound healing can be minimized with judicious patient selection and modification of the IMA harvesting method whereby a 'skeletonized' rather than 'pedicled' fashion preserves collaterals and sternal blood supply [25] and improves wound healing, particularly in diabetics. No difference has been found in myocardial enzymes [28], parameters of respiratory exchange [29] or in respiratory injury between SIMA and BIMA patients. BIMA harvesting adds around 30 minutes to a three-hour operation.

Uncertainty remains because there is no randomised evidence, therefore a randomised trial - the Arterial Revascularisation Trial (ART) - has been designed to compare SIMA versus BIMA grafting in coronary revascularisation. ART will compare survival rates, need for redo CABG, other clinical events, quality of life and cost effectiveness of SIMA versus BIMA grafting.

The main objective of ART is to assess whether the use of both IMA during CABG (BIMA) improves survival and reduces the need for further interventions over that observed with a single IMA (SIMA).

\section{Methods/Design \\ Trial design}

Two-arm, randomised multi-centre trial. Patients will be randomised to SIMA or BIMA with supplemental vein or radial artery grafts as required.

\section{Eligibility}

Inclusion criteria

- CABG patients with multi-vessel coronary artery disease (including urgent and off pump CABG patients)

\section{Exclusion criteria}

- Single graft

- Redo CABG
- Evolving myocardial infarction

- Concomitant valve surgery

\section{Randomisation and enrolment process}

All patients requiring CABG should be considered for entry into the study. Centres should keep a screening log and record if the patient is entered into the study and if not the reason why not. Patients who meet the eligibility criteria and give written informed consent should be randomised.

Randomisation will be performed by telephone contact with the trial co-ordinating centre (the Clinical Trials and Evaluation Unit (CTEU) based at the Royal Brompton Hospital in London). The randomisation service will be available 09:00-17:00 (UK time). Centres will be asked for a few simple details about the patient including initials, date of birth and eligibility criteria. The caller will be given the procedure allocation (SIMA or BIMA) and a fax will be sent to the centre confirming this.

Eligible patients will be randomised in equal proportions between the two surgical strategies SIMA or BIMA. The allocated procedure will be performed by a trial nominated surgeon who has been approved by the Trial Steering Committee as being sufficiently experienced (ie. performed $>50$ BIMA procedures).

Randomisation will be stratified by centre with specific tables using block allocation to provide treatment distribution in equal proportions. The block size will itself be subject to random variation.

To reduce the possibility of outcome measure events occurring after randomisation and before revascularisation, surgery should be performed within 6 weeks of randomisation.

\section{Surgical procedure}

It is left to the individual centres to decide the optimal anaesthetic technique and method of myocardial protection for each patient. As there is a consistent mortality of around $2.5 \%$ for CABG across most UK centres this suggests that minor differences in anaesthetic technique and methods of myocardial protection do not have a major influence on perioperative mortality. Surgical details will be recorded in the case report form (CRF). The only requirement is that the surgeon follows the randomisation allocation given for the patient (ie. SIMA or BIMA).

Surgery can be performed with cardiopulmonary bypass or as an off-pump procedure according to the experience of the surgeon. 
The following surgical procedures should be applied depending on the allocation to SIMA or BIMA:-

SIMA - standard operation using SIMA to LAD plus supplemental vein or radial artery graft

BIMA - both IMA placed to left sided coronary arteries with supplemental vein or radial artery. The IMA grafts can be used as composite grafts to each other, as long as one remains in situ.

Possible combinations include

- LIMA to OM and RIMA to LAD

- LIMA to LAD and RIMA to OM (via transverse sinus)

- LIMA to LAD and RIMA as composite graft to OM

Some typical examples of SIMA and BIMA grafts are shown in Figure 1.

Please note that anastomosis of an IMA graft to the right coronary artery is an exclusion criteria (because of evidence of inferior long-term patency).

\section{Outcome measures}

Primary

- Death from any cause (cardiovascular and non-cardiovascular mortality).

This outcome will be recorded using the flagging system of the Office of National Statistics (using name, address, date of birth and NHS number).

\section{Secondary}

Secondary clinical outcome measures will be assessed in a blinded fashion by the Clinical Events Review Committee. These outcomes will be measured in hospital, at routine 6-week clinical follow-up and from telephone questionnaires. Outcomes are as follows:-

a. Cause-specific death

- Death will be classified into cardiac, other vascular and non-cardiovascular, where possible, using autopsy reports and death certificates. Death will also be classified by ICD code.

- Death due to cardiac causes: Cardiac causes of death such as congestive heart failure, arrhythmia or myocardial infarction.

- Other vascular causes of death: Vascular causes of death such as pulmonary embolus, dissection, cerebrovascular accident or bleeding event.
- Non-cardiovascular causes of death: This includes any other cause of death.

b. 30 day mortality

- Death from any cause by 30 days post surgery

- Cause-specific death by 30 days post surgery

c. 90 day mortality

- Death from any cause by 90 days post surgery

- Cause-specific death by 90 days post surgery

d. Need for re-intervention

ie percutaneous coronary intervention or redo surgery

e. Other clinical events

- Myocardial infarction, major bleeding, cerebrovascular accident and other serious adverse events will be captured.

f. Quality of Life and cost effectiveness evaluation

- These outcomes will be measured from questionnaires (Rose -shortened form, EuroQol-5D, SF36 and Health Services Resource use). These outcomes will be assessed blind to the knowledge of which arm the trial the patient is in.

\section{Follow-up}

A summary of the follow up is shown in Table 1.

Annual Quality of Life questionnaires (Table 2) will be sent to study participants by post, no clinic visits are required apart from the routine clinical 6 -week post operative visit. Participants will be sent stamped addressed envelopes to improve the return rates of postal questionnaires. Study co-ordinators will telephone participants to alert them to the questionnaires arrival and to ask them about medications, adverse events and health services resource use.

\section{Health service research issues}

Information will be collected in each centre on resources used during the hospital stay, time in operating theatre, total blood and coagulant product use, time in cardiac recovery unit, days on ward; treatment of complications (eg return to theatre), drugs prescribed at hospital discharge, use of cardiac rehabilitation.

Information on subsequent in-patient episodes (including interventions and duration) on outpatient visits and diagnostic procedures, and on general practitioner and practice nurse consultations will be obtained from the patient during the annual telephone call. This call will also ask about specified medications (eg aspirin, statins, ace-inhibitors, beta-blockers, calcium channel antago- 
Table I: Follow-up schedule

\begin{tabular}{|c|c|c|c|c|c|}
\hline & Pre-op & Intra-op & Pre-discharge & 6 wk clinic visit $( \pm 1 \mathrm{~m})$ & $\begin{array}{l}\text { Annual telephone and postal } \\
\text { follow up for } 10 \text { years }( \pm 1 \mathrm{~m})\end{array}$ \\
\hline Baseline characteristics & $\sqrt{ }$ & & & & \\
\hline Clinical history & $\sqrt{ }$ & & & & \\
\hline Physical exam & $\sqrt{ }$ & & & $\sqrt{ }$ & \\
\hline Medications & $\sqrt{ }$ & & $\sqrt{ }$ & $\sqrt{ }$ & $\sqrt{ }$ \\
\hline LV function assessment & $\sqrt{ }$ & & & & \\
\hline $\begin{array}{l}\text { Quality of Life } \\
\text { assessment }\end{array}$ & $\sqrt{ }$ & & & & $\sqrt{ }$ \\
\hline $\begin{array}{l}\text { Health Service } \\
\text { Resource use }\end{array}$ & & $\sqrt{ }$ & $\sqrt{ }$ & $\sqrt{ }$ & $\sqrt{ }$ \\
\hline Operation details & & $\sqrt{ }$ & & & \\
\hline Clinical Outcomes & & & $\sqrt{ }$ & $\sqrt{ }$ & $\sqrt{ }$ \\
\hline
\end{tabular}

nists). Participating centres will also record and report subsequent rehospitalisations and revascularisations of patients in the trial.

\section{Trial size}

\section{Number of patients}

To achieve an absolute 5\% reduction in 10-year mortality (ie from $25 \%$ to $20 \%$ ), with $90 \%$ power at $5 \%$ alpha requires 2928 patients. The mortality data is taken from a summary of previous studies published in reference 12 .

The aim is to enrol at least 3000 patients (1500 in each arm) over a 2 to 3 year recruitment period in 20 centres in the UK, Australia, Poland and Brazil (Table 3). As the intervention is the operation, compliance is likely to be $100 \%$ except in the unusual situation where the planned operation is not possible for technical reasons.

Loss to follow-up

- For the primary outcome (ie death of trial patients) there should be minimal loss as patient death will be automatically flagged via the Office of National Statistics

- For secondary outcome measures around 5\% of patients may be lost to follow-up due to non-compliance with questionnaires or movement to other areas. Study coordinators at each centre will maintain telephone contact with participants to record Health Service Resource Use, to record adverse events, to alert them of the question- naires' arrival, to ensure addresses are current and to follow-up any non-responders.

\section{Statistical issues}

Type of analyses

The trial data will be analysed on an intention-to-treat basis with patients included in the groups assigned at randomisation, irrespective of future management and events. Outcome measure event tracking will begin at randomisation and continue until death or the end of followup. Analysis of time to event data will use log rank tests and possibly other methods suitable for survival data, in particular to take account of known prognostic variables.

\section{Frequency of analyses}

A limited number of interim analyses will be performed by the trial statistician as specified by the Data Monitoring Committee (DMC). The accumulating results will not be available to the trialists or other principal investigators. The final, definitive analysis of the trial data will be conducted 120 months after the date of commencement of the trial.

\section{Analyses of cost-effectiveness and quality of life data}

Overall analysis will be performed from the perspective of the health care system; basic information will also be collected on employment status during follow-up from the annual Health Service Resource use questionnaire. Unit costs obtained from centres and from national sources

Table 2: Quality of Life questionnaires

\begin{tabular}{|c|c|c|c|c|c|c|c|c|c|c|c|c|}
\hline \multirow[t]{2}{*}{ Questionnaire } & \multirow[t]{2}{*}{ Information collected } & \multicolumn{11}{|l|}{ YEAR } \\
\hline & & Pre-op & I & 2 & 3 & 4 & 5 & 6 & 7 & 8 & 9 & 10 \\
\hline Rose (shortened form) & Simple, evaluates angina & + & + & + & + & + & + & + & + & + & + & + \\
\hline EuroQuol-5D & Short and simple, for economic evaluation & + & + & + & + & + & + & + & + & + & + & + \\
\hline SF-36 & Detailed, examines eight separate dimensions & + & & & & & + & & & & & + \\
\hline
\end{tabular}


Table 3: ART Principal Investigators and centres

\begin{tabular}{l}
\hline UK centres \\
Mr A Forsyth, Royal Sussex County Hospital, Brighton \\
Mr A Ritchie, Papworth Hospital, Cambridge \\
Mr P O'Keefe, University Hospital Of Wales, Cardiff \\
Mr V Zamvar, Edinburgh Royal Infirmary, Edinburgh \\
Mr A Cale, Castle Hill Hospital, Hull \\
Mr T Spyt, Glenfield Hospital, Leicester \\
Mr W Dihmis, Cardiothoracic Centre, Liverpool \\
Mr J Gaer, Harefield Hospital, London \\
Mr J Desai, King's College Hospital, London \\
Professor J Pepper, Royal Brompton Hospital, London \\
Mr V Chandrasekaran, St. George's Hospital, London \\
Mr R Hasan, Manchester Royal Infirmary, Manchester \\
Mr S Clark, Freeman Hospital, Newcastle \\
Professor D Taggart, John Radcliffe Hospital, Oxford \\
Mr N Briffa, Northern General Hospital, Sheffield \\
Australian centre \\
Professor B Buxton, Austin \& Repatriation Medical Centre, Victoria \\
Polish centres \\
Professor A Bochenek, Ist Dept of Cardiac Surgery, Katowice \\
Professor S Wos, 2nd Dept of Cardiac Surgery, Katowice \\
Dr R Pawlaczyk, Medical University of Gdansk, Gdansk \\
Brazilian centre \\
Dr F Moraes, Heart Institute of Pernambuco, Recife
\end{tabular}

will be used to obtain a cost per patient. Missing data will be handled via multiple imputations in statistical analyses.

In line with the primary outcome of the trial, the main outcome measure in the cost-effectiveness analysis will be life years gained. These will be estimated within-trial and over the patients' lifetime, by taking the sum of life years obtained in each arm of the trial within the follow-up period and modelling subsequent life expectancy, using different assumptions about any within-trial treatment effects continuing. The cost per quality adjusted life year (QALY) will be calculated in the same way, using results from the EQ-5D annual questionnaires.

The cost-effectiveness analysis will be reported in terms of the incremental cost per life year and the incremental cost per QALY gained. All resource use, cost, outcome and cost-effectiveness information will be reported as the mean per patient in each arm of the trial and the mean difference, with appropriate measures of variance. Costeffectiveness in sub-groups will be estimated by applying any overall relative risk reduction and cost reduction to different baseline absolute risk groups. Cost-effectiveness acceptability curves and net benefit statistics will also be reported.

Sub-group analyses

i. Diabetic vs nondiabetic ii. Age $>70$ years vs age $<70$ years

iii. On-pump vs off-pump

iv. Radial vs vein grafts

v. Number of grafts

vi. Left ventricular failure

\section{Trial organisation}

Trial Steering Committee (TSC)

The main role of the TSC is to monitor and supervise the progress of the trial. The TSC membership is listed in Table 4. Independent members are Professor Vermes (patient lay member), Professor Sleight (Chairman and cardiologist), Dr Stables (cardiologist), Ms Farrell (trials advisor). The TSC will meet prior to the start of the trial and then annually or as required thereafter.

\section{Data Monitoring Committee (DMC)}

The main role of the DMC is to consider the data from any interim analyses and specifically to assess any safety issues (such as unexpected serious adverse events) that occur and report back to the TSC. The DMC membership is listed in Table 5. All members of the DMC are independent of the trial. The DMC will meet at the start of the trial and then annually or as required thereafter. The DMC will be expected to develop, in agreement with the investigators, a charter outlining their responsibilities and operational details.

\section{Clinical Event Review Committee}

The Clinical Event Review Committee will review adverse events during the study and adjudicate them to ensure the events meet the definitions given. Each event will be independently adjudicated by two committee members. A third committee member will be called to adjudicate an event if agreement is not reached by two members. These assessments will be blind to the knowledge of which arm of the trial the patients are in.

\section{Study co-ordination}

The study will be co-ordinated and managed by the Clinical Trials and Evaluation Unit (CTEU) a dedicated clinical trials department within the Royal Brompton Hospital. In addition to providing overall project co-ordination, the CTEU will assist in preparing the final proto$\mathrm{col}$, the investigators' manuals, design the Case Report Forms (CRF), provide the randomisation service and design and instigate the data management system. The CTEU will ensure that the trial runs according to the preagreed timetable, recruitment targets are met, CRFs are completed accurately and that all aspects of the study are performed to the highest quality. The CTEU will also assist 
Table 4: Trial Steering Committee membership

\begin{tabular}{|c|c|c|c|}
\hline Name & Trial Role & Title & Affiliation \\
\hline Professor G Vermes & Patient Lay Member & $\begin{array}{l}\text { Emeritus Professor of Hebrew } \\
\text { Studies }\end{array}$ & Oxford \\
\hline Professor D Altman & Statistician & Professor of Statistics in Medicine, & Oxford \\
\hline Professor J Dark & Lead Surgeon & Professor of Cardiac Surgery & Newcastle \\
\hline Ms B Farrell & Trials Advisor & $\begin{array}{l}\text { Co-Director, Resource Centre for } \\
\text { Randomised Trials }\end{array}$ & Oxford \\
\hline Dr M Flather & Co-Principal Investigator & $\begin{array}{l}\text { Director, CTEU, Royal Brompton } \\
\text { Hospital }\end{array}$ & London \\
\hline Professor A Gray & Health Economist & Professor of Health Economics & Oxford \\
\hline Professor J Pepper & Lead Surgeon & Professor of Cardiac Surgery & London \\
\hline Professor P Sleight & CHAIRMAN & Emeritus Professor Cardiology & Oxford \\
\hline Professor K Channon & Cardiologist & $\begin{array}{l}\text { Professor of Cardiovascular } \\
\text { Surgery }\end{array}$ & Oxford \\
\hline Dr R Stables & Independent Cardiologist & Consultant Cardiologist & Liverpool \\
\hline Professor D Taggart & Chief Investigator & Consultant Cardiac Surgeon & Oxford \\
\hline
\end{tabular}

in the training of investigators and co-ordinators at the start-up of the study and for performing monitoring procedures throughout.

The trial management team from CTEU will meet the Chief Investigator weekly by conference call.

\section{Centres}

Study sites

The list of participating centres is given in Table 3. Each centre will be paid a pro-rata sum of 1.0 FTE for years 12, 0.5 FTE for year 3 and 0.2 FTE for years 4-10, to help support a study co-ordinator.

Study site co-ordinators will be responsible for screening patients (and recording the data on a screening log), enrolling patients into the trial, providing a contact point for patients, liasing with CTEU, completing CRFs, ensuring annual questionnaires are sent and processed, recording adverse events, ensuring forms are sent to CTEU and that all edit queries are resolved.

\section{Data collection}

Each centre will be provided with a Protocol, Manual of Operations, questionnaires and patient CRFs. Data will be recorded onto two part NCR CRFs and the top copy sent to the CTEU at the times specified. Specific adverse event forms for death, myocardial infarction, major bleed, cerebrovascular accident, revascularisation and other serious adverse events (ie. other events that require or prolong hospitalisation) are provided. Centres are required to complete these adverse event forms and fax to the CTEU within 72 hours of their knowledge of the event.

\section{Investigators' responsibilities}

Surgeons must have completed a minimum of 50 BIMA operations before commencing on the study. Investigators must ensure that Local Ethics Committee approval has been obtained as well as Agreements signed off by their Institution prior to the start of the study.

Investigators are required to ensure compliance to the protocol, CRFs and Manual of Operations. Investigators are required to allow access to study documentation or source data on request for monitoring visits and audits performed by the CTEU or any regulatory authorities.

\section{Training}

\section{Pre-study training visit}

Before the study commences each centre will receive a training visit by CTEU. These visits will ensure that personnel at each site (including principal investigators, coinvestigators and the study site co-ordinator) fully understand the protocol, CRF and the practical procedures for the study.

\section{Monitoring visits}

At regular intervals during the study CTEU will perform monitoring visits to each centre. The purpose of these visits is to ensure compliance to the protocol and that ethical and regulatory guidelines are met. Source data verification and checking of essential documents will be performed. Monitoring visits also provide an opportunity for further training if required (eg new staff). Central review of study data will also be performed throughout the study.

\section{Close-out visit}

At the end of the study each centre will receive a site visit from CTEU to resolve any outstanding edit queries or adverse events and to verify the correct storage of study documentation. 
Table 5: Data Monitoring Committee membership

\begin{tabular}{lll}
\hline Name & Trial Role & Title \\
\hline Professor S Yusuf & CHAIRMAN & Consultant Cardiologist \\
Professor S Pocock & Statistician & Professor of Medical Statistics \\
Professor D Julian & Cardiology advisor & Emeritus Professor of Cardiology London \\
Professor T Treasure & Surgical advisor & Professor of Cardiothoracic \\
& & Surgery \\
\hline
\end{tabular}

\section{Ethics}

This study will conform to the MRC Guidelines for Good Clinical Practice in Clinical Trials (1998) [30] and the Declaration of Helsinki guidelines (2004) [31]. The study protocol will be submitted to the appropriate Ethical Review Committee in each country participating in the study and approval will be obtained before the study commences.

\section{Informed consent}

"Informed consent" requires individual discussion with the patient about the nature of the procedures to be conducted in a language that is easy to comprehend. The patient should fully understand that he/she might be allocated to either the SIMA or the BIMA group. The patient should also understand that his/her refusal to participate in the study will not affect the quality of subsequent medical care. It is recommended that, if possible, the patient has at least 24 hours to think about participation and discussing with family or friends before signing the consent form.

Before any trial-related procedures may be performed, informed consent must be obtained from the patient by the investigator by means of a signed declaration. The investigator must sign in the CRF to confirm that informed consent was obtained and store the original of the signed declaration of consent in the patient's notes. A copy should be given to the patient and a copy filed in the patient's CRF.

\section{Publication policy}

The results from the trial will be submitted for publication in a major journal irrespective of the outcome. The Trial Steering Committee will be responsible for approval of all manuscripts arising from the study prior to submission for publication. Sub-studies of centre-specific data may only be carried out with the knowledge and approval of the Trial Steering Committee.

Authorship of presentations and reports related to the study will be in the name of the collaborative group. The final follow-up study results paper will name local coordinators as well as those involved in central co-ordination and trial management. Co-ordinators who provided data will be named. Certificates of collaboration will be provided to those who have made a substantial contribution but whose name is not on the final report.

Papers on other aspects of the study will be published with those who made substantive contributions being named as authors. These papers will make appropriate acknowledgement of the contribution of the collaborativegroup. At the end of the study, patients will be able to request a copy of the results of the study from the investigator at that site.

\section{Proposed timetable for ART study}

Jun 2004 Grant awarded by MRC \& BHF - Recruitment starts

Jun 2007 Recruitment completed

2009 First patient completes 5 years

2012 Last patient completes 5 years - Analysis and publication of 5 year data

2014 First patient completes 10 years

2017 Last patient completes 10 years

2017/8 Publication of 10 year data

\section{Discussion}

The ART study is one of the first randomised trials to evaluate the effects on survival and other clinical outcomes of SIMA grafting versus BIMA grafting, and will help to establish the best approach for patients requiring CABG surgery.

\section{Abbreviations}

CABG: coronary artery bypass graft

IMA: internal mammary artery

SIMA: single internal mammary artery

BIMA: bilateral internal mammary artery 
OPCAB: off-pump coronary artery bypass

LIMA: left internal mammary artery

RIMA: right internal mammary artery

LAD: left anterior descending artery

OM: obtuse marginal branch of the circumflex coronary artery

CRF: case report form

\section{Competing interests}

The author(s) declare that they have no competing interests.

\section{Authors' contributions}

DPT: conceived of the study and participated in the design and co-ordination and helped to draft the manuscript

BL: participated in the design and co-ordination and helped to draft the manuscript

AG: participated in the design and the health economic analysis and helped to draft the manuscript

DA: participated in the design and statistical analysis helped to draft the manuscript

MF: participated in the design and helped to draft the manuscript

KC: participated in the design and helped to draft the manuscript

All authors read and approved the final manuscript.

\section{Study support}

ART is funded jointly by a grant from the British Heart Foundation (SP/03/001) and a grant from the Medical Research Council (G0200390). The BHF and MRC peerreviewed the grant application and have approved the manuscript submission.

\section{Source of funding for authors}

Each author is supported directly by their host institution.

\section{Acknowledgements}

Jill Mollison and Ed Juszczak for statistical support - funded by ART study grant and NHS R\&D

Oliver Rivero-Arias and Andrew Briggs for health economic analysis funded by ART study grant and University of Oxford
Fiona Nugara for data management - funded by ART study grant and Royal Brompton Hospital

Pauline Newlands for database design - funded by ART study grant and

Royal Brompton Hospital

\section{References}

I. Yusuf S, Zucker D, Peduzzi P, et al.: Effect of CABG surgery on survival: overview of 10 -year results from randomised trials by the CABG Trialists Collaboration. Lancet 1994, 344:563-570.

2. Keogh BE, Kinsman R: National Adult Cardiac Surgical Database Report 1999-200I.

3. Bucher HC, Hengstler P, Schindler C, et al:: PTCA versus medical treatment for non-acute coronary heart disease: meta-analysis of randomised controlled trials. BMJ 200I, 321:73-7.

4. Loop FD, Lytle BW, Cosgrove DM, et al.: Influence of the internal mammary artery graft on 10 -year survival and other cardiac events. N Engl J Med I986, 3 |4: I-6.

5. Cameron A, Davis KB, Green G, et al.: Coronary bypass surgery with internal-thoracic-artery grafts: effects on survival over a I5-year period. $N$ Engl J Med 1996, 334:216-219.

6. Fitzgibbon GM, Kafka HB, Leach HA, et al.: Coronary bypass graft fate and patient outcome: angiographic follow-up of 5,065 grafts related to survival in reoperation in 1,388 patients during 25 years. J Am Coll Cardiol 1996, 28:616-626.

7. Eagle KA, Guyton RA, Davidoff R, et al.: ACC/AHA Guidelines for CABG surgery: executive summary and recommendations. Circulation 1999, 100: 1464-1480.

8. Weintraub WS, Jones EL, Craver JM, Guyton RA: Frequency of repeat coronary bypass or coronary angioplasty after CABG using saphenous vein grafts. Am J Cardiol I994, 73:103-I I2.

9. Kaul TK, Fields BL, Wyatt DA, et al.: Reoperative coronary artery bypass surgery: early and late results and management in 1300 patients. J Thorac Cardiovasc Surg 1995, 36:303-312.

10. Fremes SE, Levington C, Naylor CD, et al.: Optimal antithrombotic therapy following aortocoronary artery bypass: a meta-analysis. Eur J Cardiothorac Surg 1993, 7:169-180.

II. The Post CABG Trial Investigators: The effect of aggressive lowering of low-density lipoprotein cholesterol levels and lowdose anticoagulation on obstructive changes in saphenousvein coronary-artery bypass grafts. N Engl J Med 1997, 336:153-62.

12. Taggart DP, D'Amico R, Altman DG: The effect of arterial revascularization on survival: a systematic review of studies comparing bilateral and single internal mammary arteries. Lancet 200I, 358:870-5.

13. Lytle BW, Blackstone EH, Loop FD, et al.: Two internal thoracic artery grafts are better than one. J Thorac Cardiovasc Surg 1999, I 1 7:855-872.

14. Endo $M$, Nishida $H$, Tomizawa $Y$, Kasanuki $H$ : Benefit of bilateral over single internal mammary artery grafts for multiple coronary artery bypass grafting. Circulation 200I, 104(18):2164-70.

15. Sergeant $P$, Blackstone E, Meyns B: Validation and interdependence with patient-variables of the influence of procedural variables on early and late survival after CABG. Eur J Cardiothorac Surg 1997, I 2: I-19.

16. Kurlansky PA, Traad EA, Galbut DL, et al.: Efficacy of single versus bilateral IMA grafting in women: a long-term study. Ann Thorac Surg 2001, 7 I (6): 1949-57. discussion 1957-8.

17. Schmidt SE, Jones JW, Thornby Jl, et al.: Improved survival with multiple left-sided bilateral internal thoracic artery grafts. Ann Thorac Surg 1997, 64:9-15.

18. Verhelst R, Etienne PY, El Khoury G, et al.: Free internal mammary artery graft in myocardial revascularization. Cardiovasc Surg 1996, 4:2 I 2-6.

19. Buxton BF, Ruengsakulrach P, Fuller J, et al.: The right ITA graftbenefits of grafting the left coronary system and native vessels with a high grade stenosis. Eur J Cardiothorac Surg 200I, 18:255-6I.

20. Calafiore AM, Contini M, Vitolla G, et al.: Bilateral internal thoracic artery grafting: long-term clinical and angiographic results of in situ versus $\mathbf{Y}$ grafts. J Thorac Cardiovasc Surg 200I, I 20(5):990-6. 
21. Dion R, Glineur D, Derouck D, et al.: Long-term clinical and angiographic follow-up of sequential internal thoracic artery grafting. Eur J Cardiothorac Surg 200I, I 7(4):407-I4.

22. Taggart DP, Westaby S: Neurological and cognitive disorders after CABG. Curr Opin Cardiol 200I, I 6(5):27I-6.

23. loannidis JP, Galanos O, Katritsis D, et al.: Early mortality and morbidity of bilateral versus single ITA revascularization: propensity and risk modeling. I Am Coll Cardiol 200I, 37(2):52I-8.

24. Matsa $M, P a z$ Y, Gurevitch J, et al.: Bilateral skeletonized internal thoracic artery grafts in patients with diabetes mellitus. J Thorac Cardiovasc Surg 200I, I 2 I (4):668-74.

25. Cohen AJ, Lockman J, Lorberboym M, et al.: Assessment of sternal vascularity with single photon emission computed tomography after harvesting of the ITA. J Thorac Cardiovasc Surg 1999, I I 8(3):496-502.

26. Gurevitch J, Paz Y, Shapira I, et al.: Routine use of bilateral skeletonized internal mammary arteries for myocardial revascularization. Ann Thorac Surg 1999, 68(2):406-I I. discussion 412

27. Wendler O, Hennen B, Markwirth T, et al: Complete arteria revascularization in the diabetic patient-early postoperative results. Thorac Cardiovasc Surg 200 I, 49(I):5-9.

28. Taggart DP: Biochemical assessment of myocardial injury after cardiac surgery: effects of a PAF antagonist, bilateral ITA grafts, and coronary endarterectomy. J Thorac Cardiovasc Surg 200I, I 20(4):65I-9.

29. Taggart DP: Respiratory dysfunction after cardiac surgery: effects of avoiding cardiopulmonary bypass and the use of BIMA. Eur J Cardiothorac Surg 200I, I 8(I):3I-7.

30. MRC Guidelines for Good Clinical Practice in Clinical Trials (1998) [http://www.mrc.ac.uk]

31. World Medical Association Declaration of Helsinki (2004) [http://www.wma.net]

Publish with Bio Med Central and every scientist can read your work free of charge

"BioMed Central will be the most significant development for disseminating the results of biomedical research in our lifetime. "

Sir Paul Nurse, Cancer Research UK

Your research papers will be:

- available free of charge to the entire biomedical community

- peer reviewed and published immediately upon acceptance

- cited in PubMed and archived on PubMed Central

- yours - you keep the copyright 